\title{
The Daniel K. Inouye Solar Telescope (DKIST)/Visible Broadband Imager (VBI)
}

\author{
Friedrich Wöger ${ }^{1} \cdot$ Thomas Rimmele $^{1}$ (D) Andrew Ferayorni ${ }^{1} \cdot$ Andrew Beard $^{1}$. \\ Brian S. Gregory ${ }^{1}$. Predrag Sekulic ${ }^{1,2}$. Steven L. Hegwer ${ }^{1}$
}

Received: 14 June 2021 / Accepted: 20 August 2021 / Published online: 4 October 2021

(c) The Author(s) 2021

\begin{abstract}
The Daniel K. Inouye Solar Telescope (DKIST) is a ground-based observatory for observations of the solar atmosphere featuring an unprecedented entrance aperture of four meters. To address its demanding scientific goals, DKIST features innovative and state-of-the-art instrument subsystems that are fully integrated with the facility and designed to be capable of operating mostly simultaneously. An important component of DKIST's first-light instrument suite is the Visible Broadband Imager (VBI). The VBI is an imaging instrument that aims to acquire images of the solar photosphere and chromosphere with high spatial resolution and high temporal cadence to investigate the to-date smallest detectable features and their dynamics in the solar atmosphere. VBI observations of unprecedented spatial resolution ultimately will be able to inform modern numerical models and thereby allow new insights into the physics of the plasma motion at the smallest scales measurable by DKIST. The VBI was designed to deliver images at various wavelengths and at the diffraction limit of DKIST. The diffraction limit is achieved by using adaptive optics in conjunction with post-facto image-reconstruction techniques to remove residual effects of the terrestrial atmosphere. The first images of the VBI demonstrate that DKIST's optical system enables diffraction-limited imaging across a large field of view of various layers in the solar atmosphere. These images allow a first glimpse at the exciting scientific discoveries that will be possible with DKIST's VBI.
\end{abstract}

Keywords Instrumentation and data management

\section{Introduction}

The Daniel K. Inouye Solar Telescope (DKIST: Rimmele et al., 2003, 2020; Keil et al., 2003a,b) is the first four-meter class telescope for observations of the Sun. Among the capabilities that DKIST offers is the ability to resolve structures in the solar photosphere, chromosphere, and corona with an unmatched spatial resolution. The Visible Broadband Imager (VBI) is an imaging instrument capable of observations at DKIST's diffraction limit in eight

This article belongs to the Topical Collection:

The Daniel K. Inouye Solar Telescope (DKIST)

Guest Editors: Thomas R. Rimmele, Philip R. Goode, Michael Knölker, Jeffrey R. Kuhn, and Robert

Rosner

Extended author information available on the last page of the article 
spectral bands corresponding to various physical layers of the solar atmosphere. VBI consists of two separate channels operating in mutually exclusive wavelength bands. The VBI blue channel typically operates between $380-550 \mathrm{~nm}$, whereas the VBI red channel covers wavelengths between $550-850 \mathrm{~nm}$. The exact wavelength band entering each channel is set by DKIST's Facility Instrument Distribution Optics (FIDO). FIDO is a suite of mirrors, windows, and dichroic beamsplitters that can be flexibly rearranged in DKIST's optical path to direct a specific chromatic portion of the solar beam into a particular instrument or instrument channel. Realistically, over one thousand scientifically valuable configurations are feasible with FIDO, including many that allow high spatial and temporal resolution observations using the VBI channels. Each of the two VBI channels is capable of switching to any one of four wavelengths within 3.2 seconds using a high-speed filter wheel, allowing the creation of time series of interleaved observations at different diagnostics of the solar atmosphere. This allows addressing many unanswered scientific questions related to details of the morphology of the plasma in the solar atmosphere and its dynamic behavior.

Aspects of the VBI are similar to several other imaging instruments at current 1-1.5 meter class solar telescopes operating in the optical-wavelength range. The Rapid Oscillations in the Solar Atmosphere (ROSA) instrument at the Dunn Solar Telescope (DST) in Sunspot, New Mexico, USA is an imaging instrument that employs multiple high-speed cameras, with the capability to operate at specific wavelengths that are fixed during the observation (Jess et al., 2010). The Swedish Solar Telescope (SST) on La Palma, Spain in its early operations routinely used imaging channels at various but fixed wavelengths to image the solar atmosphere (Scharmer et al., 2003). The Chinese New Vacuum Solar Telescope (NVST) has an imaging instrument for observations in the Fraunhofer G-band, $\mathrm{H} \alpha$, and $\mathrm{TiO}$ (Liu et al., 2014). The Broadband Imager (BBI) instrument installed at the German GREGOR telescope on Tenerife, Spain consists of a dual-imaging-channel design, where the wavelengths in the respective channel can be manually switched (von der Lühe et al., 2012). The GREGOR/BBI also provides additional modes allowing for telescope performance analysis and multiple options for image reconstruction. Similarly, the Goode Solar Telescope (GST) located at Big Bear Lake in California, USA also employs fixed imaging channels, typically operating in the $\mathrm{H} \alpha$ line (with a typically narrower Lyot filter) and the TiO band head (Goode and Cao, 2013). The VBI not only constitutes a significant improvement in terms of spatial resolution. In addition, the aforementioned examples for broadband images typically do not employ filter wheels, therefore each camera channel operates at a fixed, single wavelength. For the VBI, a fast filter wheel was developed that allows line switching within a fraction of a second, allowing quasi-simultaneous observations at wavelengths within the same channel, and strictly simultaneous observations at the wavelengths chosen in the two individual channels.

In the following, we describe examples of the scientific questions that the VBI intends to address, and we explain its design and operational features in more detail.

\section{Science Objectives and Requirements}

The VBI is an instrument capable of high-cadence imaging at the diffraction limit of DKIST. Its mission is to acquire "movies" of this quality at a number of wavelengths in the visible regime $(390-850 \mathrm{~nm})$ that correspond to scientifically valuable diagnostics in various layers of the solar atmosphere. As an imaging instrument, the VBI will use indirect detection methods to trace magnetic fields. For example, it will use the radiative properties of highly temperature-sensitive molecular bands that typically show magnetic elements in 
high contrast compared to the surrounding photosphere that exhibits weak magnetic fields. The subsections below highlight some of the scientific use cases the VBI will be capable of addressing. The DKIST Critical Science Plan provides a more comprehensive summary of DKIST use cases, many of which include the VBI either as principal instrument or in support of the spectro-polarimeters (Rast et al., 2021).

\subsection{Magneto-convection and Large Scale Magnetic Structures in the Solar Photosphere}

Our understanding of the inner structure of Sun-like stars is directly related to observations of the solar convection zone and its radiative layer, known as the photosphere. Computational models have gotten quite sophisticated and detailed in their attempts to simulate the mechanisms of magnetic-field generation, its evolution, dispersion, and how it connects to the upper layers in the solar atmosphere (Stein and Nordlund, 1998, 2006; Wedemeyer et al., 2004; Vögler et al., 2005; Moll, Cameron, and Schüssler, 2011). Even though many advances have been made to verify these models with both ground-based and satellite imaging instruments there are still unanswered questions, for example: how do persistent, strong magnetic elements - that we know exist and can be traced using imaging data - form and develop over time (Hinode Review Team et al., 2019), or how does vortex motion ("tornadoes") (Wedemeyer-Böhm et al., 2012) in the photosphere contribute to the coronal heating? In particular the latter question has recently spurred intense research because the discovered vortex motion is hypothesized to play an important role for the transport of energy from the photospheric layer into the chromosphere and corona (e.g. Yadav, Cameron, and Solanki, 2021). The VBI's capabilities are specifically targeted at imaging the solar photospheric flow fields and magnetic structures at the highest possible spatial and temporal resolution available from the DKIST, enabling comparisons of their interactions with magnetic elements with those of current state-of-the-art numerical simulations of magneto-convection.

Advances in numerical modeling of sunspots have revealed that sunspots are highly structured formations with convective elements inside of the umbra ("umbral dots"), varying magnetic fields across the umbra, highly dynamic flows and variations of angles within the penumbra, and "light bridges" that often demarcate fracture lines on which sunspots eventually break up. As with the magneto-convective flows in the photosphere, some of the new insights about sunspot structure have come from magnetohydrodynamic numerical simulations (Cheung et al., 2010; Bharti, Beeck, and Schüssler, 2010; Rempel, 2012). Using the GREGOR broadband imager, Schlichenmaier et al. (2016) were able to support findings of intricate differences in the substructure ("dark lanes") in light bridges between models and observations. Analysis of observed images at the resolution limit of DKIST should provide even deeper insights into the fundamental physical processes in the solar photosphere that are believed to occur on scales smaller than $50 \mathrm{~km}$. More recently, using ROSA imaging data among others, Jess et al. (2020) studied the oscillatory behavior above sunspot umbrae and penumbrae and found evidence for an acoustic resonator above the sunspot that would explain the previously observed amplification of magnetohydrodynamic wave amplitudes as they propagate through the solar atmosphere in that region. With its synchronization and cadence requirements, the VBI will be able to contribute to oscillation studies with unprecedented spatial resolution.

\subsection{Chromospheric Dynamics}

The convective processes in the solar atmosphere generate acoustic waves with different frequencies. Waves generated below the photosphere with frequencies below the acoustic 
cutoff frequency at about $5 \mathrm{mHz}$ are reflected in the gravitationally stratified solar atmosphere and are trapped below, while higher-frequency waves are able to travel upwards into the chromosphere. Numerical simulations indicate that these waves form shocks in the lowdensity chromosphere and contribute to its heating (e.g. Carlsson and Stein, 1997; Wedemeyer et al., 2004). How much mechanical heating is contributed, where it is deposited, and how much wave energy is generated are still a topics of intense research. Possibly related are spicules that are surmised to act as waveguides, be connected to the transport of energy from the photosphere into the chromosphere and corona (Tsiropoula et al., 2012), and even accelerate the solar wind (De Pontieu et al., 2007). With DKIST's maximum spatial resolution, the VBI will provide high-cadence data that will allow new insights into the physical parameters and morphological properties of spicules to answer questions about formation and underlying photospheric conditions.

The chromosphere is known to be highly dynamic in general, but the explosive dynamics of solar flares requires particularly high temporal resolution. It is commonly believed that solar flares represent a process of rapid transformation of the magnetic energy of active regions into the kinetic energy of energetic particles and plasma flows, and heat. High-resolution, hard X-ray and microwave observations have shown that flare bursts occur in finely structured elementary bursts on time scales from tens of milliseconds to a few seconds (Benz, 2017). Other questions revolve around the signature of flares at continuum wavelengths ("white light flares") and the location of the deposition of the energy that causes them. It is surmised that often the intensity "continuum enhancement" is underestimated owing to the fact that instruments do not resolve the bright flare footpoint kernels (Kleint et al., 2016).

Elementary bursts need to be studied at higher spatial resolution in the visible to obtain complementary data on their physical properties. The VBI will be able to take high-cadence observations in chromospheric lines, like Ca II K (393.3 nm) and $\mathrm{H} \alpha$, and photospheric continua simultaneously and at the highest resolution achievable with the DKIST. This will allow monitoring the reaction of both the chromosphere and the photosphere to the fast reorganization of the magnetic field and the corresponding heating phenomena at very small spatial and short temporal scales. The VBI will also serve as the context instrument for spectro-polarimetric measurements of flare regions, providing both higher cadence and a larger field of view than the DKIST Visible Spectro-Polarimeter (VISP), Diffraction-Limited Near-Infrared Spectro-Polarimeter (DL-NIRSP), and Visible Tunable Filter (VTF).

\subsection{Coronal Observations}

The detailed mechanism for energy deposition into and the heating of the solar corona continues to be an active research topic. The corona is believed to be controlled by the magnetic field dominating the tenuous and highly ionized plasma (e.g. Fan and Gibson, 2004). High-cadence observations at high angular resolution near the limb of the Sun will support analysis of the dynamics and morphology of the corona in its many different structures like prominences and their cavities, active-region loops, and open-field regions. The VBI will be able to acquire Fe XI (789.2 nm) images simultaneous with either photospheric or chromospheric diagnostics to investigate the connection between these atmospheric layers of the Sun. While the VBI is not optimized for such observations, DKIST's capabilities such as the 2.8 arcminute limb occulter with integrated active tracking perpendicular to the limb will improve such observations. The VBI will also provide the context to spectro-polarimetric measurements as they are provided by, for example, the DL-NIRSP. 
Table 1 Summary of the VBI high level optical design requirements.

\begin{tabular}{lll}
\hline & Requirement & Comments \\
\hline Spectral coverage & $390-860 \mathrm{~nm}$ & \\
Field of view & $\varnothing 2 \operatorname{arcmin}$ circular $(\mathrm{H} \alpha, \mathrm{H} \beta)$ & $\begin{array}{l}\text { circular for the narrowest } \\
\text { bandwidth filters }\end{array}$ \\
& $2 \times 2 \operatorname{arcmin}^{2}$ square $($ all others $)$ & max. static wavefront error \\
Static aberrations & known static aberrations with & contribution, correctable \\
& peak-to-valley amplitude $<\lambda / 2$, & using image processing \\
for Zernike polynomials higher & than focus & VBI blue channel \\
Spatial sampling & Nyquist-Shannon at $430.5 \mathrm{~nm}$ & VBI red channel \\
Relative photometry & Nyquist-Shannon at $656.3 \mathrm{~nm}$ & \\
Signal-to-Noise ratio & $2 \times 10^{-2}$ of continuum intensity & \\
Instrument location & in final image & \\
\hline
\end{tabular}

\section{Design Rationales and Overview}

The VBI addresses certain scientific requirements by providing two independent camera channels that are conceptually identical but are optimized for two exclusive wavelength ranges. The VBI "blue" channel operates in the wavelength range from 390-550 nm, whereas the VBI "red" operates in the wavelength range from 550-860 nm.

Already at its inception, the VBI was planned to coordinate with various DKIST subsystems (Wöger et al., 2010, 2012; McBride et al., 2012). This includes optical systems such as DKIST's Wavefront-Correction System (WFC) and FIDO, but also software systems such as the instrument control system and many others. In this section, we describe the design of the VBI, and the reasoning behind some of the more significant design choices.

\subsection{Optical Design}

\subsubsection{High-Level Requirements}

The optical design of the VBI was guided by requirements that were set based on the scientific questions outlined in Section 2. They are summarized in Table 1. The requirements for the optical design ensure that the VBI has the capability to deliver images with a resolution at DKIST's diffraction limit for most wavelengths within its spectral range. This extends to requirements that the VBI benefit from an adaptive-optics corrected beam, and furthermore that single acquired VBI frames have a signal-to-noise ratio - approximated (under the assumption of being photon-noise limited) as SNR $=\bar{S} / \sqrt{\bar{S}}=\sqrt{\bar{S}}$, where $\bar{S}$ is the signal mean - sufficient for the application of image-reconstruction algorithms (see also Section 3.2.3). The latter effectively sets a throughput requirement for the VBI optics.

The VBI optical-design tolerances were specified to achieve a nominal Strehl ratio of about $90 \%$ across the full field of view in more than $80 \%$ of configurations incurred in a Monte-Carlo analysis of possible configurations. This helped us arrive at feasible manufacturing tolerances for all optical and opto-mechanical components. Figure 1 shows the layout of the instrument on the optical tables installed at the DKIST facility. The design concept of the VBI is such that most requirements can be met simultaneously, i.e. meeting 


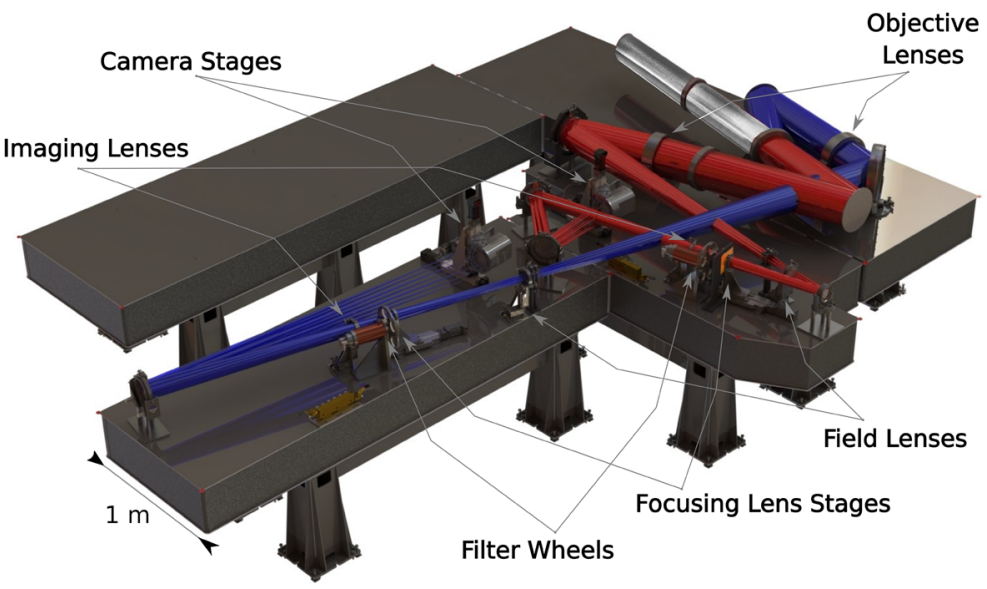

Figure 1 The layout of the VBI on the DKIST coudé floor. On the top, the beam (light-gray rays) begins where the DKIST 2.8 arcminute beam is reflected off the deformable mirror of the DKIST/WFC subsystem. Approximately $96 \%$ of the light is passed through the first refractive component, the beamsplitter deflecting about $4 \%$ of the light into the optical path of the wavefront sensors. Subsequently, a short-wavelength part of the beam is deflected into the VBI "blue" channel with a dichroic beamsplitter (blue rays), while the remaining red part is transferred into the VBI "red" channel (red rays). The fold mirrors, lenses, high-speed filter wheels, camera stages, and cameras are depicted in their final configuration as they exist on the optical tables in the DKIST coudé laboratory. Courtesy of L. Rimmele.

one requirement will not inhibit other requirements from being met, which has resulted in each channel receiving individual optimizations. For example, in order to ensure the highest possible photon throughput, each optic received a custom anti-reflective coating that is particularly effective within the spectral range of its channel. In the following, we describe in more detail how the optical design achieves some of its most important requirements.

Both VBI channels follow a similar, simple refractive design approach to arrive at an optimal image quality for each wavelength (see Figure 2). The optical interface for both channels is the location of the last preceding dichroic beamsplitter part of FIDO. For each channel the beam is directed to a custom-built objective lens $(\varnothing \approx 28 \mathrm{~cm})$ that creates a focal plane near the field lens, which relays the beam to the focusing lens that creates a collimated pupil at the location of the interference filter. In the VBI red channel, an otherwise difficultto-compensate aberration within the objective lens caused by imperfections in the manufacturing process is removed by means of a thin phase plate between the focusing lens and the filter wheel. The phase plate was manufactured based on interferometric measurements of the objective lens' deviations from the design wavefront, and incorporates the inverse aberrations in order to "null" the induced wavefront error that otherwise would have caused a significant reduction in the achievable image quality. The pupil on the filters in both channels has a diameter of 65 millimeters; particular care was taken to align the filters perpendicular to the beam to minimize the field-dependent shift of the transmitted wavelength towards shorter wavelengths (see Section 3.1.2). The filtered beam subsequently passes through the imaging lens that forms a focused field on the sensor in the camera plane. The VBI employs several opto-mechanical motion stages: the linear focusing stage that moves the collimator lens to focus the beam for each wavelength, the filter wheel to switch interference filters, and linear stages to move the camera for field sampling (see Section 3.1.4).

The final optical alignment at the summit yielded a spatial sampling of approximately 0.011 arcseconds pixel ${ }^{-1}$ in the VBI blue channel, and approximately 0.016 arcsec- 


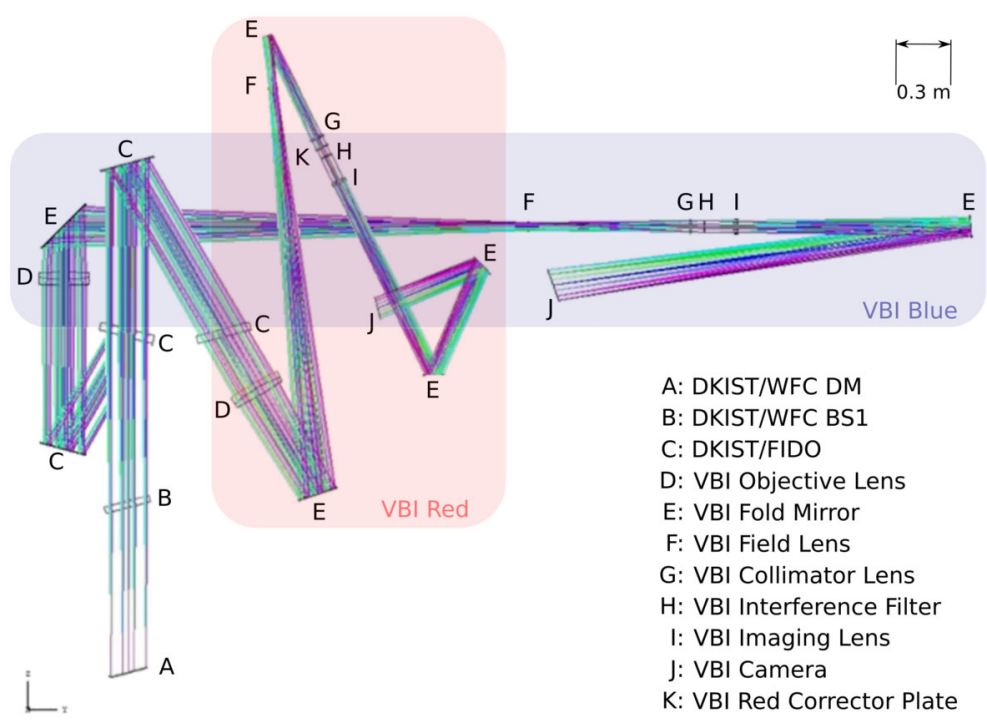

Figure 2 Optical layout of the VBI. The VBI blue channel's approximate footprint is shaded in blue, the VBI red channel's in red. Internally, i.e. starting at the objective lenses, each channel of the VBI has a beam path length of approximately $4 \mathrm{~m}$.

onds pixel $^{-1}$ in the VBI red channel, slightly oversampling the diffraction limit. The final residual wavefront error within each instrument channel has been measured interferometrically through the instrument's internal optical elements, and it is better than $\lambda / 15$ RMS at each channel's field center. As reference, the corresponding Strehl value of 0.979 is very close to the design value of 0.988 for the VBI red channel's reference wavelength of $656 \mathrm{~nm}$. The VBI blue channel is performing slightly less well with a Strehl of 0.953 (design: 0.98) at the reference wavelength $430 \mathrm{~nm}$. These values indicate that the VBI is - as intended by the requirements - only a very small contributor to the overall DKIST, wavefront error budget that considers errors for all telescope optics as well as residual atmospheric wavefront error. In reality, for a large, ground-based facility such as the DKIST the limiting factor for the quality of single raw VBI images will almost without exception be the residual atmospheric wavefront error after adaptive-optics correction.

\subsubsection{Interference Filters}

As mentioned, each of the two VBI channels is optimized for its own operating wavelength regime; in total the VBI meets its spectral-range requirement with the combination of both channels. The VBI employs interference filters to select narrow wavelength bands within its spectral range that correspond to particular scientific diagnostics in the solar atmosphere. Within its specific spectral range, each channel has the capability to observe any diagnostic if the corresponding interference filter is available $(\varnothing 70 \mathrm{~mm})$. A list of the first available interference filter characteristics is given in Table 2. The interference filters were individually tested at the Dunn Solar Telescope in Sunspot, NM to ensure that the required central wavelength and bandpass requirements were met by the vendor (Wöger, 2014).

The interference filters are located in a high-speed, custom-built filter wheel in a collimated beam at a pupil plane to minimize the diameter of the optic. Each filter wheel is capable of switching to a new position well within the required $533 \mathrm{~ms}$ : the physical move 
Table 2 Available diagnostics in the VBI "blue" (top half) and the VBI "red" (bottom half). The shown represent results measured at the Dunn Solar Telescope.

\begin{tabular}{llll}
\hline Diagnostic & Atm. region & $\begin{array}{l}\text { Central } \\
\text { Wavelength [nm] }\end{array}$ & $\begin{array}{l}\text { FWHM } \\
{[\mathrm{nm}]}\end{array}$ \\
\hline Ca II K & chromosphere & 393.327 & 0.101 \\
G-band & photosphere & 430.52 & 0.437 \\
blue cont. & photosphere & 450.287 & 0.406 \\
$\mathrm{H} \beta$ & chromosphere & 486.139 & 0.046 \\
$\mathrm{H} \alpha$ & chromosphere & 656.289 & 0.047 \\
red cont. & photosphere & 668.423 & 0.442 \\
TiO & photosphere & 705.839 & 0.578 \\
Fe XI & photosphere/corona & 789.186 & 0.350 \\
\hline
\end{tabular}

time to an opposite position on the wheel can be achieved in about $300 \mathrm{~ms}$, while subsequent settling prior to the begin of an exposure requires less than an additional $80 \mathrm{~ms}$ in a tuned filter wheel. The filter wheel can be optionally cooled. In the standard-use case of one movement every 3.2 seconds the filter wheel does not heat up so the cooling lines have not been hooked up to the DKIST facility coolant by default. Whenever more experimental use cases are executed, the filter wheel can heat up and will require coolant flow. The VBI filter wheel meets the interface standard of the DKIST facility for thermal control so that fluid can easily be provided in those exceptional cases.

When positioning an interference filter in a collimated beam of an extended source, the image near the field edges will show a wavelength shift towards shorter wavelengths because the rays corresponding to these field points arrive at an angle on the filter. In a fully collimated beam, the shift towards shorter wavelengths can be described by

$$
\lambda_{\theta}=\lambda_{0} \sqrt{1-\left(\frac{n_{\mathrm{ext}}}{n_{\mathrm{eff}}}\right)^{2} \sin ^{2}(\theta)},
$$

where $\lambda_{\theta}$ is the shifted wavelength at the angle of incidence $\theta, \lambda_{0}$ is the wavelength at an angle of incidence of zero, $n_{\mathrm{ext}}$ is the refractive index of the surrounding material (air), and $n_{\mathrm{eff}}$ is the effective refractive index of the filter. Typically, $n_{\mathrm{eff}} \approx 1.9$ or higher for VBI's interference filters. The optical design of the VBI is such that $\theta$ at the filter location within each channel is controlled to lead to an incurred blue shift at the corner of the required field less than half of the full width at half maximum (FWHM) bandpass of each filter. With the filters with the narrowest bandpass, i.e. the $\mathrm{H} \beta$ and $\mathrm{H} \alpha$ filters with a FWHM of less than $0.05 \mathrm{~nm}$, being the most critical, a compromise had to be struck between the manufacturing tolerances for homogeneity, and the clear-aperture size that drives the wavelength shift. A shift of less than half of the FWHM bandpass can be tolerated without a significant change of the morphological chromospheric scene; Figure 3 shows at the example of the $\mathrm{H} \alpha$ absorption line how the "worst case" projected shift towards shorter wavelengths relates to the profile of the absorption line itself.

\subsubsection{Detectors}

The state-of-the-art CMOS sensors, developed by ANDOR for the VBI, that feature $4128 \times$ 4104 sensor pixels which are $12 \mu \mathrm{m}$ square, have a full-well capacity of greater than $40 \mathrm{~K}$ 


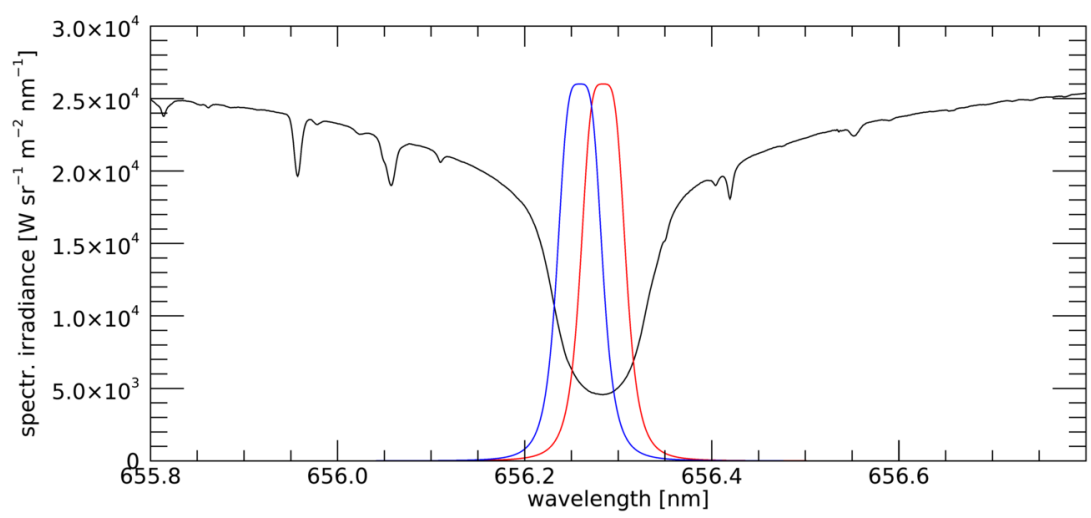

Figure 3 Profile of the $\mathrm{H} \alpha$ absorption line, with the simulated transmission profile of the filter in the center of the field of view (red) and at its edge (blue).

photo-electrons and a quantum efficiency higher than $60 \%$ at the wavelength of $600 \mathrm{~nm}$. These specifications are sufficient for the VBI to achieve its signal-to-noise ratio requirement given all other constraints such as, e.g., the fixed sampling. After calibration the sensors show a linearity of better than $0.1 \%$ over the full-well capacity. The sensor is protected by a wedged and anti-reflection coated entrance window that avoids the creation of fringes due to etalon effects. The camera has been demonstrated to have an exposure trigger jitter of about $8 \mu \mathrm{s}$, sufficient for the VBI's internal synchronization needs and even those of DKIST instruments that have to synchronize to a polarization modulator. Both VBI cameras can be synchronized using DKIST's Time Reference and Distribution System (TRADS) to sub-millisecond accuracy, exceeding VBI's requirement of $\pm 5 \mathrm{~ms}$ by a large margin, and allowing precise measurements of, e.g., oscillations at two scientific diagnostics simultaneously (see Section 3.2.1).

The camera has two readout modes that can be used interchangeably. In "global shutter" mode the camera reads out all pixels simultaneously ensuring that all pixels were exposed at the same time, whereas in "rolling shutter" mode the camera reads the sensor out row by row so that the first row read out was exposed at an earlier time than the last row read out. This allows us to expose the camera at frame rates of around $30 \mathrm{~Hz}$ and higher. However, the two readout modes have different noise characteristics. In particular the "global shutter" mode exhibits on average a fixed spatial frequency noise pattern at very specific spatial frequencies that exceeds the noise floor at all other spatial frequencies by about a factor of two hundred. This spatial frequency pattern is related to the CMOS readout electronics in the sensor. The noise floor is reduced in the "rolling shutter" mode of the camera where correlated double sampling (in-pixel removal of an offset by acquisition and subtraction of a reference measurement) can be employed, and with it there is a reduction of the fixed-frequency noise pattern. If not properly attenuated in a pre-processing step, this noise is amplified in the image-reconstruction process rendering the reconstructed images of limited use for scientific analysis because in particular high spatial frequencies - and hence, structures close to the diffraction limit - are affected. For this reason, the VBI - while it has the capability to use the camera in both readout modes - currently defaults to the "rolling shutter" mode even though the "global shutter" mode is more favorable for image-reconstruction purposes (see Section 3.2.3). Changing the default readout mode in normal operations is currently only planned under special circumstances. 

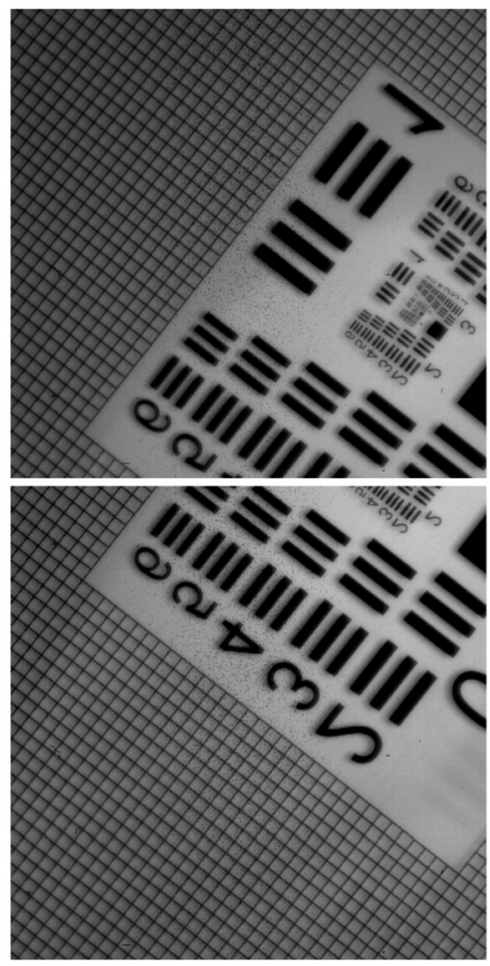
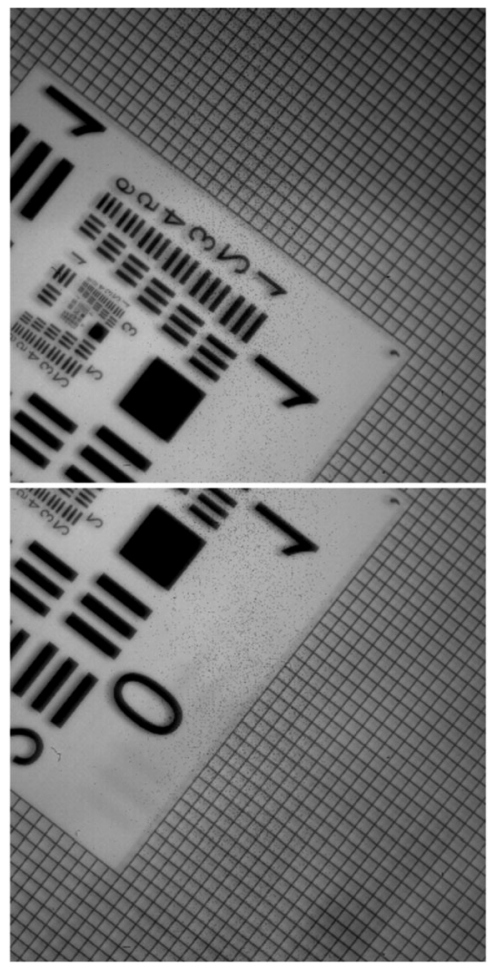

Figure 4 First commissioning image of the Air Force Resolution Target located in the secondary focus of DKIST, using the field-sampling mode of the VBI red. Each grid square has an edge length of $0.5 \mathrm{~mm}$.

\subsubsection{Field Sampling}

In order to achieve the full field of view of $2 \times 2$ arcminutes $^{2}$ that DKIST provides for all of the post-adaptive-optics instruments while also adhering to its sampling requirements, the VBI uses the largest-format fast detectors available today (see Section 3.1.3). However, these detectors are still not large enough to acquire the full field in a single image: to sample the full field using Nyquist-Shannon sampling at $430.5 \mathrm{~nm}$ would require a sensor with more than $10^{8}$ pixels. A scientific sensor of that size that operates at a frame rate of $30 \mathrm{~Hz}$ is not readily available. Given the requirements for image reconstruction, a frame rate of $30 \mathrm{~Hz}$ or greater is a key requirement (see Section 3.2.3). Finally, the combination of size and frame-rate requirements to acquire the full field in a single image would result in a to-date prohibitive bandwidth requirement to handle the data stream.

For these reasons the VBI samples the full field of view with multiple positions. The VBI camera is mounted on motion stages and moves in the two directions perpendicular to the beam-propagation direction (see Figure 1), with a field overlap that is at minimum 1.5 arcseconds but typically much larger (see Figure 4). To sample the full field using the VBI red channel $2 \times 2$ field positions are needed, each requiring 3.2 seconds. This means that approximately 13 seconds are required to acquire the four mosaic positions, each imagereconstructed position representing 2.67 seconds evolution of the solar atmosphere. Therefore, even sub-sonic evolution of features within the time interval of the first and last field position acquisition are detectable given the VBI red channels' very fine spatial sampling. 
This makes the assembly of the full field of view potentially challenging and feature blurring may occur in the overlap regions. Current plans foresee the provision of individual, non-assembled field position images but including accurate metadata denoting the overlap. This will allow scientific analysis of the data without compromised spatial resolution.

To maintain simplicity and flexibility of the optical design and ensure repeatability of the corresponding motions, the VBI achieves the field sampling by employing high-resolution linear motion stages. Additional benefits to this solution are reduced complexity of opticalalignment procedures and configuration on the optical table, as well as the fact that the VBI optics will be capable of accommodating future large and fast sensors without any modification once this technology becomes available. The precision encoders allow positioning of the camera to an absolute accuracy of less than a quarter of a pixel, sufficient to accurately recombine the field-sample images to a single frame at a later time, i.e. the recombination accuracy is only limited by feature evolution on the Sun. The movement from one field position to an adjacent field position is performed within 380 milliseconds including settle time of the stages. Hence, they can be and are synchronized with filter-wheel motions whenever necessary to maximize the VBI's data-acquisition efficiency. The stage motors have been sized so that they do not significantly heat up in continuous operation.

\subsection{Software Components and Integration}

\subsubsection{VBI Integrated Software}

The VBI software integrates into and makes use of the framework provided by DKIST's high level software (HLS). The primary systems used are

i) Common Services Framework (CSF) The DKIST/CSF provides a base framework for software control and communication between components. Most major software systems of DKIST - in particular, all of the following ones - use CSF for easy integration into the Observatory Control System. CSF provides software controllers for hardware components commonly used in DKIST subsystems, such as WFC and VBI (Hubbard, Goodrich, and Wampler, 2010). The provision of a common software infrastructure is meant to reduce software development and maintenance efforts.

ii) Observatory Control System (OCS) The DKIST/OCS is the software system facing the personnel who control and operate the telescope. All major DKIST subsystems provide graphical user interfaces (GUI) that are imported into the OCS, allowing its user to directly control them using a single access point (Tritschler et al., 2016). The VBI GUI will - just like all other instruments - be accessible via the OCS.

iii) Instrument Control System (ICS) The DKIST/ICS provides a thin interface between the OCS and the instruments, allowing for status checking of and coordination between instruments (Johansson and Goodrich, 2012). The VBI GUI is imported into the OCS through the ICS.

iv) Data Handling System (DHS) The DKIST/DHS provides the infrastructure - both hardand software - to transport the "raw" camera data, and process and store them at high speeds. Given the camera hardware capabilities (Section 3.1.3), the DHS has to be capable of transporting multiple $960 \mathrm{MiB} \mathrm{s}^{-1}$ data streams simultaneously (Wampler and Goodrich, 2009; Cowan and Wampler, 2011). The VBI uses the DHS plugin infrastructure to, e.g., calibrate and reconstruct 80 short-exposed image frames into a single output image facilitating speckle image-reconstruction algorithms. Finally, the DHS is responsible for gathering and combining the acquired data with their metadata, and delivering the final product to the DKIST Data Center (Goodrich, Wampler, and Hubbard, 2008). 
v) Camera Systems Software (CSS) The DKIST/CSS provides a common interface to the DKIST camera hardware. It abstracts the low-level control into a common interface for the different detectors employed at DKIST. It provides robust access to typical camera functionality and simplifies future maintenance of the instrument software. The VBI uses the CSS and TRADS to synchronize its exposures with its motion stages while attaching vital metadata to each frame necessary for later calibration.

vi) Time Reference and Distribution System (TRADS) TRADS is a vital tool used throughout the DKIST facility to synchronize subsystems at any level with each other. It uses the Precision Time Protocol (PTP, IEEE-1588) to deliver an absolute time signal based on the Global Positioning System time to any subsystem within DKIST that requires it (Ferayorni et al., 2014). Within the DKIST subsystems TRADS is capable of synchronizing hardware to about tens of nanosecond relative accuracy to an absolute time. All instrument subsystems that require precise synchronization between their components, such as the VBI that has to synchronize its cameras to fast-moving motion stages, utilize TRADS to achieve accurately timed exposures.

The VBI was the first instrument to integrate, and integrate into, these DKIST provided software components and has contributed significantly to developing the software into a robust and functional product.

\subsubsection{VBI Control Software}

The VBI control software integrates the DKIST/CSF as the underlying framework for interacting with its hardware and other DKIST subsystems. It makes use of, and has contributed to, the improvement of CSF's Standard Instrument Framework (SIF), which provides common software components for instrument control (Ferayorni, 2012).

The overarching control scheme for all DKIST instruments is time-based and in the majority of the cases, such as the one for the VBI, it relies on repeatable moves and exposures executed at exact times. The DKIST project has encouraged collaboration and use of common control hardware by providing CSF compliant top-level interfaces to selected controllers. An example of the collaboration between the DKIST instrument and software teams is the VBI implementation of the high-level interface to the hardware controllers. The stringent timing specifications of the VBI - any motion has to complete and settle within 533 $\mathrm{ms}$ - require the VBI to employ real-time hardware controllers that are pre-programmed to perform their motion within a specific time window at a particular absolute time. A $32 \mathrm{kHz}$ pulse synchronized to the absolute reference time distributed by the DKIST/TRADS subsystem then triggers the execution of the motion-control program as well as the start of the camera exposures. Through characterization of settle times and monitoring of the encoder positions, this allows time-efficient control of the instrument while ensuring that the motion has finished punctually. This implementation has been integrated into the CSF SIF and adopted by multiple instruments (Ferayorni et al., 2014).

\subsubsection{Real-Time Image Reconstruction}

The VBI uses the DKIST/DHS pipeline processing capabilities to post-facto reconstruct a number of acquired, short-exposed images (typically around 80 frames) that freeze the distortions introduced by Earth's atmosphere into a single, high spatial resolution image. The reconstruction algorithm is based on well-understood technology: speckle interferometry for extended sources. The particular core algorithm used, the "Kiepenheuer-Institut Speckle Interferometry Package" (KISIP), has been implemented for use of extended sources in the 
past (Wöger, von der Lühe, and Reardon, 2008), and it is widely accepted and used at several solar telescopes world wide such as the DST (e.g. Jess et al., 2010), GREGOR (von der Lühe et al., 2012), and the GST (Goode and Cao, 2013). Nonetheless, the algorithm used in the VBI image-reconstruction pipeline has been re-implemented from scratch, and it was modified to make use of general-purpose graphics processing units (Wöger et al., 2010; Wöger and Ferayorni, 2012). As a result, it has been improved to achieve high photometric precision in its reconstructions (Peck, Wöger, and Marino, 2017). The choice to perform speckle image reconstruction in real-time at the site of the telescope is founded on the recognition of two key aspects:

i) The speckle interferometric algorithm is computationally expensive but can be programmed to exploit parallelism in the calculation. This makes it feasible to arrive at real-time reconstruction performance, even for $4 \mathrm{k} \times 4 \mathrm{k}$ images. In case of the VBI, 80 short-exposed frames are acquired within 2.67 seconds (at $30 \mathrm{~Hz}$ ). Subsequent, synchronized and simultaneous motion-stage movements can occur and settle within $533 \mathrm{~ms}$, giving the VBI 3.2 seconds to reconstruct the frames into a single $4128 \times 4104$ pixel image. This gives summit personnel near real-time, high spatial resolution feedback related to the scene on the Sun at various atmospheric layers.

ii) Continuously acquiring full-size data frames at $30 \mathrm{~Hz}$ results in a data stream with a bandwidth of $960 \mathrm{MiB} \mathrm{s}^{-1}$. Assuming a four-hour observation, the necessary data storage size is of the order of $10 \mathrm{TiB} \approx 11 \mathrm{~TB}\left(1 \mathrm{TiB}=1024^{4}\right.$ bytes $)$. Reconstructing images on-summit reduces the data volume needed to be transported to the DKIST Data Center by a factor of 80: a large reduction for the required bandwidth and long term storage.

The image data are ingested by a VBI provided DHS processing-plugin that has the capability to pre-process the raw data. Pre-processing involves real-time dark compensation, pixel gain correction, and optional frame selection prior to beginning the speckle image reconstruction. If frame selection is desired prior to the reconstruction process, an increase of the number of acquired images to select at a minimum 80 frames from is required resulting in an overall decrease of the cadence at which reconstructions are generated. The raw frames are selected using either one of two metrics: contrast in image $I$ (defined as $C_{I}=\sigma_{I} / \bar{I}$ ) or total absolute image gradient. Which algorithm is used in the process is decided well in advance of the observation, with the default being the "total absolute gradient" algorithm that appears to yield slightly better results. The input 80 (or more) frames will be used to compute the bispectrum that encodes the object's Fourier phases for each of a large number of overlapping tiles of typically $128 \times 128$ pixels. Therefore, the tile size corresponds to a field of view of about $1.5 \times 1.5$ arcseconds $^{2}-$ well within the size of a typical isoplanatic patch, which is the field angle over which the atmospheric point spread function can be assumed to be constant. The Fourier phases for each tile are subsequently recovered from the bispectrum using recursive and iterative phase-recovery algorithms.

In parallel with the image data, the VBI speckle image-reconstruction pipeline ingests the control data of the DKIST/WFC system. These data are used to determine the prevailing seeing conditions, the Fried parameter $r_{0}$, in real-time, as well as to estimate the performance of the system during image-data acquisition. Recent studies have shown that precise knowledge of the $r_{0}$-parameter is key to photometrically accurate reconstructions (Peck, Wöger, and Marino, 2017). In order to obtain an estimate of $r_{0}$ using the WFC system control data, each control data frame is represented as a series of Karhunen-Loève modal coefficients. Both pre- and post-correction modal coefficients can be extracted from a complete set of the WFC control-system data that encode information on the wavefront correction currently applied 
and the measurement of the residual wavefront error. The sum of pre- and post-correction modes constitutes the instantaneous wavefront distortion present at that moment in the telescope optical system, and a highly dynamic variation is caused primarily by atmospheric turbulence. It can be shown that for Kolmogorov turbulence, the (temporal) variance of each atmospheric Karhunen-Loève mode coefficient is proportional to $\left(D / \mathrm{r}_{0}\right)^{5 / 3}$, where $D$ represents the diameter of the telescope (Wang and Markey, 1978). Using pre-computed model proportionality factors based on the Kolmogorov assumption, Karhunen-Loève modal coefficient variances measured within the VBI's 2.67 second data-acquisition interval can be fitted using $\left(D / \mathrm{r}_{0}\right)^{5 / 3}$ as a free parameter, yielding a robust estimate of the $\mathrm{r}_{0}$-value during the acquisition interval. The per-mode ratio of residual vs. atmospheric modal variance can be used to estimate the correction performance, which is a necessary input for the computation of the calibration functions (Wöger and von der Lühe, 2007; Peck, Wöger, and Marino, 2017).

Two entities are important for the calibration of the Fourier amplitudes, which is a vital step in ensuring precise relative photometric properties in the reconstructed images: The spectral ratio - approximated within each tile image burst as $\epsilon(\boldsymbol{q})=|\overline{i(\boldsymbol{q})}|^{2} / \overline{\left(\left.i(\boldsymbol{q})\right|^{2}\right.}$, where $i(\boldsymbol{q})$ is the Fourier transform of image intensity at the spatial frequency $\boldsymbol{q}$ (von der Lühe, 1984) - encodes the combined effect of atmospheric seeing $\left[\mathrm{r}_{0}\right]$ and WFC system performance on the images. The speckle transfer function represents the attenuation of the average of the modulus squared of the Fourier amplitudes that are used in the speckle reconstruction process. This attenuation needs to be reversed in order to arrive at a precise estimate of the object's photometric properties. Theoretical model functions for both the speckle transfer functions and the spectral ratios were pre-computed for a large range of values for $\mathrm{r}_{0}$, modal-correction performance, and field angles, and they can be looked up in an extensive look-up table that is pre-loaded into memory at startup of the instrument. As part of the image-reconstruction process, the modulus squared of the Fourier amplitudes in each tile are averaged over all input frames and subsequently calibrated using a speckle transfer function appropriate for the computed value of $r_{0}$ and measured spectral ratio. Once the tile speckle transfer function has been applied, the Fourier amplitudes are recombined with the recovered Fourier phases. The resulting reconstructed tile images are subsequently assembled into a full frame that is then stored for transport to the DKIST Data Center.

The reconstruction is performed on a compute server that is integrated into the DHS compute infrastructure and is accessible through the DHS software framework. The compute server incorporates ten NVIDIA ${ }^{\circledR}$ Tesla $^{\mathrm{TM}}$ V100 GPU Computing Accelerator generalpurpose graphical processing units (GPGPUs) that perform the major portion of the computation in parallel (Beard, Cowan, and Ferayorni, 2014; Beard, Wöger, and Ferayorni, 2020). The VBI provides the processing plugin software that interfaces with the DKIST/DHS software and performs the individual steps laid out above within less than 3.2 seconds.

\section{Modes of Operation}

\subsection{Data Acquisition}

The VBI allows acquisition of data in multiple ways to address a large variety of scientific use cases. Integer pixel binning, up to $4 \times 4$, can be selected for data acquisition allowing for adjustment of the signal-to-noise ratio in each pixel. Furthermore, the VBI supports the selection of a region of interest allowing for an increase of the frame rate. These options are supported in combination with frame selection. Since the VBI channels sample the full 


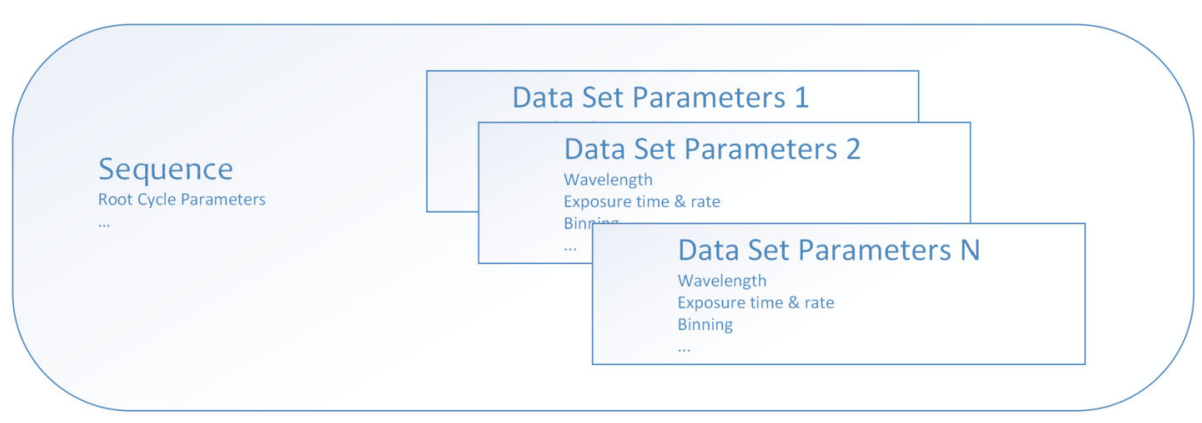

Figure 5 How DKIST instrument data acquisition, in particular also the VBI's, is programmed. Data set parameters (DSPs) encode the minimal set of settings required to acquire a particular data product, e.g. a number of images at a particular wavelength. Multiple DSPs can be sequenced through, guided by further parameters.

field of view by motion of the camera in the focal plane (see Section 3.1.4), the number of field-samples is limited to a single position whenever a region of interest is selected, because the necessary overlap to assemble the full field of view cannot be guaranteed. Additional adjustments can be made in regard to the camera rate that can be reduced to increase exposure times, allowing for adjustment of the signal-to-noise ratio while potentially reducing the cadence of the images.

Despite the many benefits of processing the images on-summit, the VBI allows for exporting unprocessed single frames in certain non-standard situations. This mechanism may prove useful for specific scientific use cases, e.g. for investigations of the temporal evolution of a flare. In this case, the highly dynamic nature of the phenomenon may benefit from each individual image in the high-cadence image sequence. However, due to limitations related to the data volume that needs to be handled, single frames can only be collected, processed, and stored for a limited time interval.

The VBI, like all DKIST instruments, organizes its data-acquisition programs in compartmentalized "atomic" Data Set Parameters (DSP) units that contain all necessary parameters to acquire a coherent set of frames (see Figure 5). For the VBI, these DSP units can primarily be differentiated by the wavelength that is to be observed. However, they also encode all other parameters, e.g. exposure time and frame rate, and selected field sampling, binning, etc. More subtle parameters such as what algorithm to use for frame selection (if applicable) are also part of a DSP unit. The DSP units can be organized in a root cycle that can be looped, facilitating the repeated acquisition of images at specific wavelengths with the same settings. Sub-cycles within the root cycle are also supported allowing the creation of arbitrarily complex data-acquisition sequences.

\subsection{Synchronization Modes}

The VBI allows multiple ways of synchronizing exposures and exposure sequences within its two channels with each other. Aside from not synchronizing the two channels at all or only at the start of the first exposure, it allows synchronization in two other ways:

i) Loose synchronization This synchronization mode allows starting each root cycle at the same time. The different DSP units within each root cycle of each channel including those within any sub-cycles start immediately after the previous one is finished. Because the number of DSP units can be different, the duration of a root cycle in each channel 


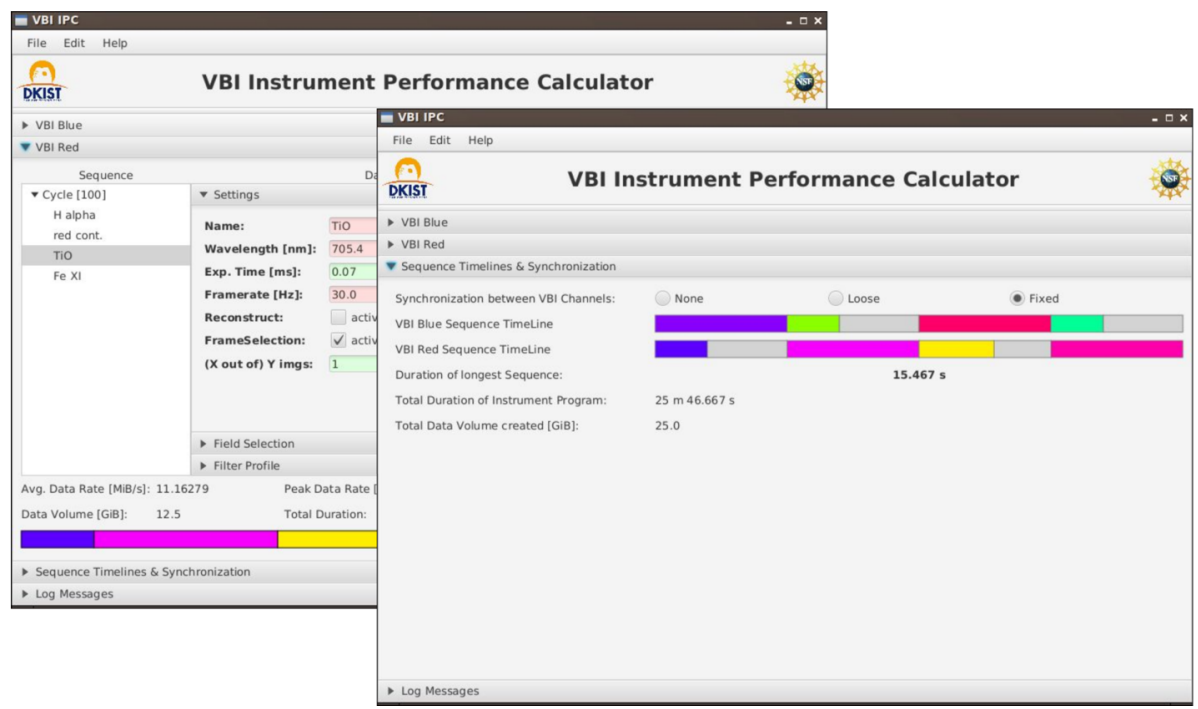

Figure 6 Screenshots of the VBI's Instrument Performance Calculator tool. The tool allows planning of simple and complex observing scenarios, including the exploration of the VBI's various modes of operation and their impact on timing, duration, and generated data volume. The image in the foreground is associated with a setup where both VBI channels are used simultaneously and synchronized in the "fixed" mode (see Section 4.2).

can be significantly different, and the channels would run asynchronously from the start. In loose-synchronization mode, a wait time is inserted for the channel that completes its root cycle first in order to re-synchronize with the other channel on the start of a new root cycle.

ii) Fixed synchronization In fixed-synchronization mode, the start of each DSP unit is synchronized with the start of a DSP unit in the other channel. This is true even if one cycle has a different number of DSP units from the other. This means that each unit is waittime padded to match the longest DSP unit in both cycles. While this mode ensures the same cadence for all DSP units, which is beneficial for certain scientific use cases such as those related to measuring oscillations, it has the potential of significantly decreasing the VBI's data-acquisition duty cycle.

These modes of operating the VBI allow the generation of data suitable for many scientific use cases including, e.g., the ones laid out in Section 2.

\subsection{Support Software for Scientists (IPC)}

The VBI team supports the creation of viable DSP unit sequences by providing a software tool that allows predicting various performance parameters related to the user input. The application can be run on any platform with an installed Java runtime. The VBI's Instrument Performance Calculator (IPC) implements the control concepts of the instrument, i.e. the codification of data acquisition using DSP units, in combination with a model for telescope and instrument throughput based on the DKIST flux budget. A user of the IPC has the ability to create arbitrarily nested cycles of DSP units to address the scientific use case. Some screenshots of the tool are shown in Figure 6. 
With this input, the IPC computes the effect of adjusting certain VBI data-acquisition modes (see Section 4) on data-acquisition times for DSP units and sequences, and it computes move duration and settle times, and an estimation for the generated data volume. The signal-to-noise ratio is computed per diagnostic based on several input parameters, including wavelength, exposure time, binning, and approximate distance from disk center. The flux at each wavelength is computed independently based on the properties of the optical surfaces as measured where measurements are available, and it uses model predictions otherwise. In particular, the solar spectral-irradiance model is based on the disk-center quiet-Sun measurements of Neckel and Labs (1984). There are currently no plans to incorporate other target types such as, e.g., sunspot umbrae, however, a model for (wavelength dependent) limb darkening was incorporated based on Neckel and Labs (1994). The modular implementation of the flux budget that is calibrated to match the DKIST project's overall flux budget allows easy adaption to the reality at the facility, making the VBI IPC more accurate. Not included in the VBI IPC are air mass and the expected FIDO configuration, for the following reasons.

The operational concepts of DKIST foresee a dynamic scheduling of experiments, based on conditions both on Earth and on the Sun. Conditions on Earth can be impacted by operational status of the instrument, current FIDO configuration, etc., but most importantly by the prevailing weather and atmospheric conditions. Solar conditions impact the scheduling of an experiment through availability of the requested target at the requested solar coordinates, or whether a target of opportunity has appeared that overrides other more common targets.

Hence, inclusion of an air-mass calculator as a tool for scientists is not reasonable as it is not possible to predict when the experiment is executed at the site. The current implementation for the flux and therefore signal-to-noise ratio is based on Zenith observations, resulting in "optimum" flux estimates.

The ability to flexibly adjust the chromatic feed for each instrument by re-configuring the FIDO dichroic-beamsplitter configuration is currently not considered in the VBI IPC. The reason is that DKIST is designed for efficient multi-instrument observations, and a tool (the FIDO Configuration Tool) to investigate the effects of the FIDO configuration on all instruments is provided by the DKIST project separately.

\section{Implementation at the DKIST Site}

The VBI was the first instrument integrated into the DKIST coudé laboratory (see Fig. 7). As the trail-blazer instrument, the VBI was also the first instrument integrated into the DKIST hardware and software infrastructure. In order to ensure efficient and timely progress, careful planning of optical integration and alignment, and the interfacing with the DKIST electrical, mechanical, thermal, and software systems was imperative. For example, an opticalalignment plan was written, toleranced, and executed in a laboratory environment well in advance of its review and execution by the DKIST optical team on the summit (Sekulic et al., 2016). Similar steps were taken for all of VBI's engineered interfaces to other DKIST subsystems (Ferayorni et al., 2016). The successful execution of these steps became part of a carefully sequenced set of the DKIST project's overall integration progression criteria.

It is important to recognize that careful planning of integration procedures is not the sole guarantor of success. The VBI's integration was deliberately phased over multiple campaigns at the site to provide sufficient time to address issues after each integration campaign. This fact was important to avoid delays due to unforeseen issues, as evidenced by, e.g., unexpected behavior of hardware at the summit. The VBI is the first instrument to employ newly designed state-of-the-art large-format detectors at full speed. While the DKIST/VBI 
Figure 7 Both VBI channels as integrated into the DKIST coudé laboratory.

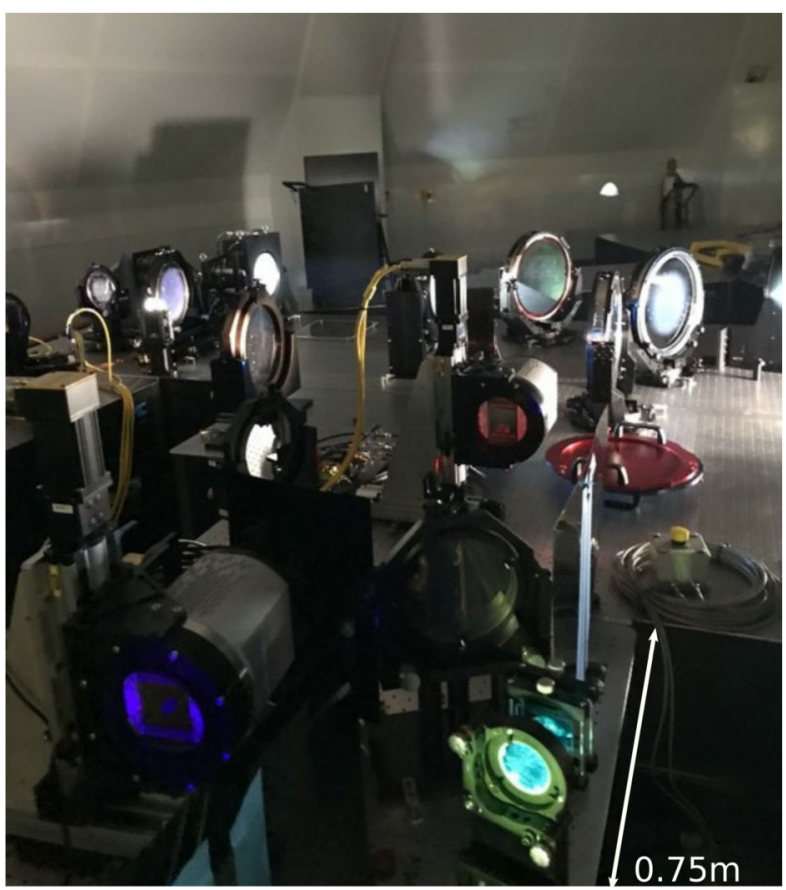

project was intimately involved in setting requirements for and the testing of these cameras, the VBI site-integration phase was the first time that the cameras were operated with the beam of a solar telescope. The phased integration of the instrument into the DKIST facility allowed adjusting to the realities of a new-to-the-market sensor being exposed to sunlight for the first time ever, and have any issues fixed before the next campaign. It also allowed early identification of further data-calibration steps that may be required for proper scientific data analysis in the future.

\section{Instrument Data}

\subsection{Data Calibration Pipeline}

The VBI provides a working prototype processing pipeline for calibration of its observed raw data. Calibration of raw frames is only necessary for data that were not image reconstructed in near real-time at the DKIST site, which is going to be the exception. This pipeline is written in the Python language. It is based on a modular approach in order to enable various tasks that can be run alone or in sequence. As part of its basic functionality, the prototype pipeline implements - at the pixel level - dark current and gain correction. In addition, the pipeline is capable of analyzing particular calibration images in order to determine the subpixel overlap between the field samples, aiding in computing an image representing the full field of view of the VBI. The VBI provided prototype pipeline has been integrated into the complex production architecture of the DKIST Data Center. 


\subsection{First-Light Initiative and First Results}

The VBI red channel acquired its first on-Sun images on 10, 11, and 12 December 2019, as part of an effort to demonstrate the optical performance of the telescope and the already integrated subsystems: DKIST's "First-Light Initiative". At that time, the VBI-provided DHS speckle-processing plugin was not finalized. For that reason, the raw data were postprocessed with a modified speckle interferometry package based on KISIP. New speckle transfer functions based on the DKIST/WFC system design and expected performance were included into the algorithms to achieve the highest possible relative photometric accuracy. During these early observations with the VBI, the only detector operating mode available was the "global shutter" mode; this mode exhibits increased noise at specific but fixed spatial frequencies (see Section 3.1.3). In order to reduce the impact of the noise on the image reconstruction, each individual, calibrated raw frame was pre-processed to attenuate the noise amplitude at these frequencies. In the following we present the first scientifically processed on-disk images in the Fe XI and TiO lines (see Table 2).

\subsubsection{Images of the Fe XI Filter}

On 10 December 2019, a limited data set was acquired using the Fe XI filter with a central wavelength of $\lambda=789 \mathrm{~nm}$. At 20:10:52 UTC, DKIST pointing was approximately (56, -16) arcseconds in helio-projective cartesian coordinates. The data were acquired with the DKIST Wavefront Correction System locked for high-order correction, under good seeing conditions $\left(\mathrm{r}_{0} \gtrsim 12 \mathrm{~cm}\right)$. The seeing conditions were such that the speckle imaging postprocessing was able to recover the full field of view and was not significantly impacted by the inherently anisoplanatic nature of DKIST's adaptive-optics correction (Figure 8). An analysis of a cross-section of a small-scale feature in the reconstructed field of view demonstrates that DKIST was operating close to the diffraction limit (Figure 9). The image of the selected elongated small-scale feature is about two pixels wide in the middle. Near the edge, it becomes a single-pixel structure with an intensity well above the local "background" intensity. The fact that approximately single-pixel structures with a FWHM of about two pixels and an intensity above the noise level can be detected shows that DKIST operated at close to the theoretical diffraction limit at of the telescope at a wavelength of $\lambda=789 \mathrm{~nm}$.

\subsubsection{Movies of $\mathrm{H} \alpha$ and TiO Intensity}

On 12 December 2019 a movie of approximately ten-minute duration was acquired with interleaved images acquired in the $\mathrm{H} \alpha$ line and the TiO band head (see Fig. 10 and Supplementary Information). The atmospheric conditions were in the range of $\mathrm{r}_{0} \approx 10 \mathrm{~cm}$, although there was a large variability. Even at slightly shorter wavelengths the individual reconstructed images show structure close to the diffraction limit of the telescope over the full acquired field of view. The time sequence has been processed using algorithms that remove remaining dynamic image distortion introduced by turbulent air motion within layers high above DKIST's entrance aperture.

\section{Conclusions and Potential for Future Upgrades}

The VBI as part of the first-light instrument suite of the DKIST project pushes the envelope of imaging instruments in several aspects, be it in regards to optical elements such as, e.g., 


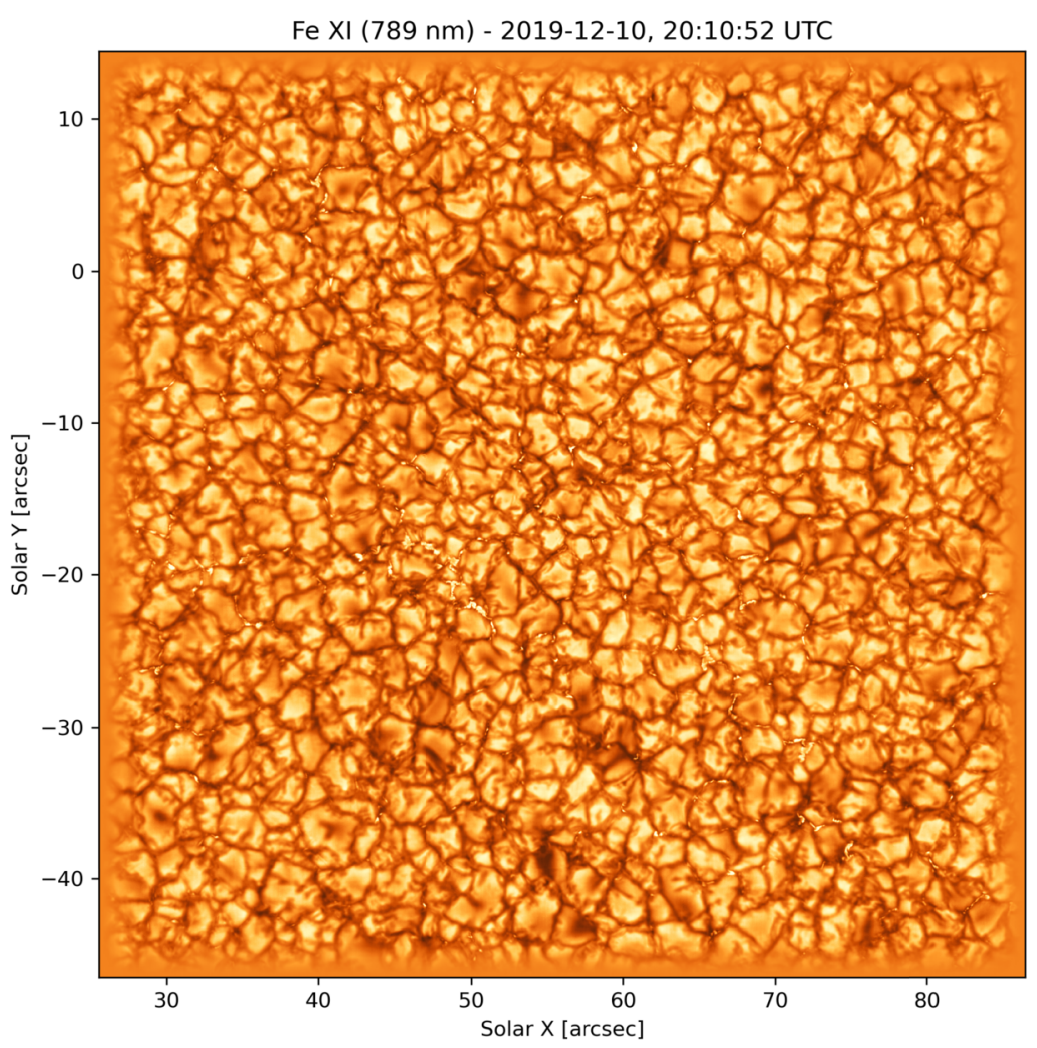

Figure 8 Speckle image reconstruction of data acquired on 10 December 2019 at 20:10:52 UTC with the VBI red channel Fe XI filter.

its narrow interference filters, or the near real-time image reconstruction of the acquired images. With its first-light data the instrument has shown it can perform at the diffraction limit set by DKIST's four-meter aperture. Its data will help address many scientific questions and offer the opportunity for many new discoveries as they provide the most detailed view of the targeted atmospheric layers in the solar atmosphere.

As the first instrument developed for and within the DKIST instrument infrastructure, the VBI has been invaluable in setting its design parameters and validating in many aspects; in doing so, the VBI has helped pave the development path for all other DKIST instruments.

Even though the already existing potential of the VBI has yet to be fully tested in the future, certain enhancements can be anticipated. The data pre-processing that is necessary to attenuate the fixed spatial frequency camera noise pattern described in Section 3.1.3 to the level of the noise floor prior to image reconstruction is being investigated, and plans foresee integration of the respective algorithms into the near-real-time speckle-processing pipeline. Those include provision of additional filters for other wavelengths in the VBI spectral range corresponding to further scientifically valuable diagnostics. Experimental coronal observations beyond the usage of the Fe XI filter can be investigated by using the "clear" position of the VBI channels to maximize photon flux and potentially facilitate their imagereconstruction capabilities. In order to achieve a better separation of chromospheric structure and reduce the amount of photospheric signal in, e.g., the $\mathrm{H} \alpha$ images, a narrower filter with a FWHM of less than $0.02 \mathrm{~nm}$ could be envisioned, although its performance across the field 

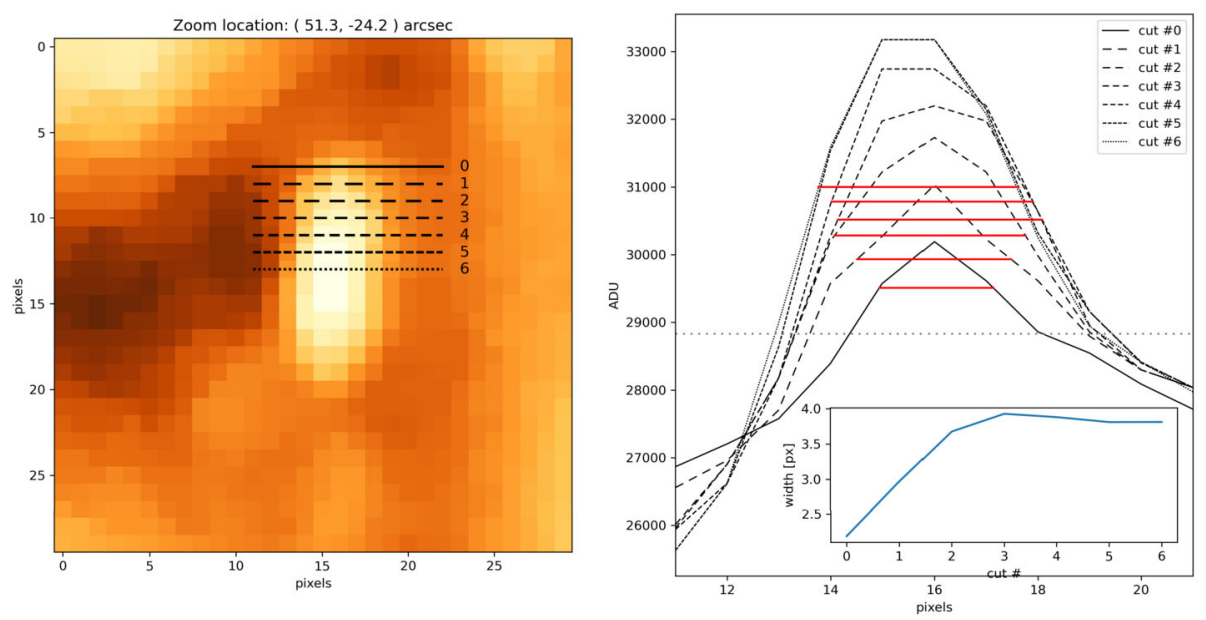

Figure 9 Left: Close-up image of the small-scale feature located at the solar helio-projective cartesian coordinates $X / Y(51.3,-24.2)$ arcseconds in the image at wavelength $\lambda=789 \mathrm{~nm}$, acquired on 10 December 2019 at 20:10:52 UTC (see Figure 8). Right: Traces of the cuts indicated in the image close-up left, demonstration that DKIST operated near the diffraction limit. The inset of the graphic displays the computed FWHM of the structure cuts, using the gray dotted horizontal line as "background" value.
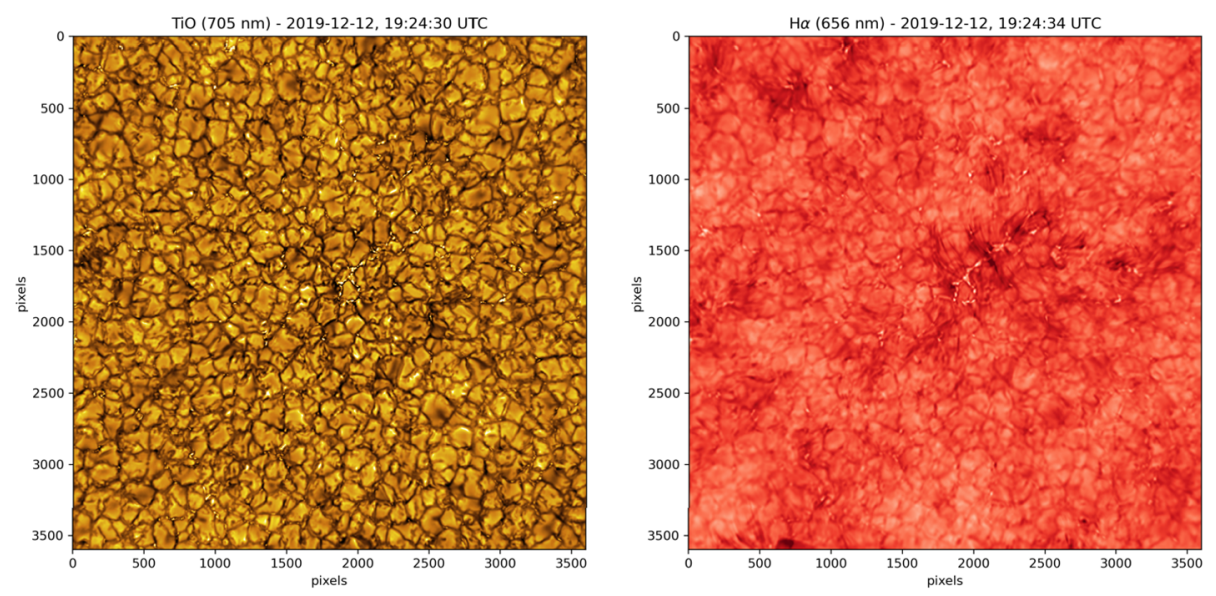

Figure 10 Left: Speckle image reconstruction of data acquired on 12 December 2019 at 19:24:30 UTC with the VBI red channel TiO filter. Right: Speckle image reconstruction of data acquired on 12 December 2019 at 19:24:34 UTC with the VBI red channel $\mathrm{H} \alpha$ filter.

of view may be impacted by the wavelength blue shift discussed in Section 3.1.2 Finally, there is an upgrade path to extend VBI functionality to operate as a broadband polarimeter if polarization optics are integrated into VBI's optical path.

Supplementary Information The online version contains supplementary material available at https://doi.org/ 10.1007/s11207-021-01881-7.

Acknowledgments The research reported herein is based in part on data collected with the Daniel K. Inouye Solar Telescope (DKIST), a facility of the National Solar Observatory (NSO). NSO is managed by the As- 
sociation of Universities for Research in Astronomy, Inc., and is funded by the National Science Foundation. Any opinions, findings, and conclusions or recommendations expressed in this publication are those of the authors and do not necessarily reflect the views of the National Science Foundation or the Association of Universities for Research in Astronomy, Inc. DKIST is located on land of spiritual and cultural significance to Native Hawaiian people. The use of this important site to further scientific knowledge is done so with appreciation and respect. The authors thank the many contributors to the VBI. These include among many others William McBride, Arthur Eigenbrot, Mihalis Mathioudakis, and Lukas Rimmele. The authors would like to thank the anonymous reviewer for very insightful comments that helped improve the manuscript.

Funding Open Access funding provided by NSO. The National Solar Observatory (NSO) is operated by the Association of Universities for Research in Astronomy, Inc. (AURA), under cooperative agreement with the National Science Foundation.

\section{Declarations}

Disclosure of Potential Conflicts of Interest The authors declare that they have no conflicts of interest.

Open Access This article is licensed under a Creative Commons Attribution 4.0 International License, which permits use, sharing, adaptation, distribution and reproduction in any medium or format, as long as you give appropriate credit to the original author(s) and the source, provide a link to the Creative Commons licence, and indicate if changes were made. The images or other third party material in this article are included in the article's Creative Commons licence, unless indicated otherwise in a credit line to the material. If material is not included in the article's Creative Commons licence and your intended use is not permitted by statutory regulation or exceeds the permitted use, you will need to obtain permission directly from the copyright holder. To view a copy of this licence, visit http://creativecommons.org/licenses/by/4.0/.

\section{References}

Beard, A., Cowan, B., Ferayorni, A.: 2014, DKIST visible broadband imager data processing pipeline. In: Chiozzi, G., Radziwill, N.M. (eds.) Software and Cyberinfrastructure for Astronomy III, Proc. Soc. Photo-Opt. Instrum. Eng. (SPIE) CS-9152, 91521J. DOI. ADS.

Beard, A., Wöger, F., Ferayorni, A.: 2020, Real-time speckle image processing with the DKIST. In: Guzman, J.C., Ibsen, J. (eds.) Software and Cyberinfrastructure for Astronomy VI, Proc. Soc. Photo-Opt. Instrum. Eng. (SPIE) CS-11452, 114521X. DOI. ADS.

Benz, A.O.: 2017, Flare observations. Liv. Rev. Solar Phys. 14, 2. DOI. ADS.

Bharti, L., Beeck, B., Schüssler, M.: 2010, Properties of simulated sunspot umbral dots. Astron. Astrophys. 510, A12. DOI. ADS.

Carlsson, M., Stein, R.F.: 1997, Formation of solar calcium H and K bright grains. Astrophys. J. 481, 500. DOI. ADS.

Cheung, M.C.M., Rempel, M., Title, A.M., Schüssler, M.: 2010, Simulation of the formation of a solar active region. Astrophys. J. 720, 233. DOI. ADS.

Cowan, B., Wampler, S.: 2011, Technologies for high speed data handling in the ATST. In: Evans, I.N., Accomazzi, A., Mink, D.J., Rots, A.H. (eds.) Astronomical Data Analysis Software and Systems XX CS-442, Astron. Soc. Pacific, San Francisco, 297. ADS.

De Pontieu, B., McIntosh, S.W., Carlsson, M., Hansteen, V.H., Tarbell, T.D., Schrijver, C.J., Title, A.M., Shine, R.A., Tsuneta, S., Katsukawa, Y., Ichimoto, K., Suematsu, Y., Shimizu, T., Nagata, S.: 2007, Chromospheric Alfvénic waves strong enough to power the solar wind. Science 318, 1574. DOI. ADS.

Fan, Y., Gibson, S.E.: 2004, Numerical simulations of three-dimensional coronal magnetic fields resulting from the emergence of twisted magnetic flux tubes. Astrophys. J. 609, 1123. DOI. ADS.

Ferayorni, A.: 2012, Instrument control software for the visible broadband imager using ATST common services framework and base. In: Radziwill, N.M., Chiozzi, G. (eds.) Software and Cyberinfrastructure for Astronomy II, Proc. Soc. Photo-Opt. Instrum. Eng. (SPIE) CS-8451, 845113. DOI. ADS.

Ferayorni, A., Beard, A., Berst, C., Goodrich, B.: 2014, DKIST controls model for synchronization of instrument cameras, polarization modulators, and mechanisms. In: Chiozzi, G., Radziwill, N.M. (eds.) Software and Cyberinfrastructure for Astronomy III, Proc. Soc. Photo-Opt. Instrum. Eng. (SPIE) CS9152, 91520Z. DOI. ADS. 
Ferayorni, A., Beard, A., Cole, W., Gregory, S., Wöeger, F.: 2016, Bottom-up laboratory testing of the DKIST visible broadband imager (VBI). In: Angeli, G.Z., Dierickx, P. (eds.) Modeling, Systems Engineering, and Project Management for Astronomy VII, Proc. Soc. Photo-Opt. Instrum. Eng. (SPIE) CS-9911, 991106. DOI. ADS.

Goode, P.R., Cao, W.: 2013, Infrared observations from the new solar telescope at Big Bear. Solar Phys. 287, 315. DOI. ADS.

Goodrich, B.D., Wampler, S.B., Hubbard, J.R.: 2008, Gathering headers in a distributed environment. In: Bridger, A., Radziwill, N.M. (eds.) Advanced Software and Control for Astronomy II, Proc. Soc. PhotoOpt. Instrum. Eng. (SPIE) CS-7019, 70192N. DOI. ADS.

Hinode Review Team, Al-Janabi, K., Antolin, P., Baker, D., Bellot Rubio, L.R., Bradley, L., Brooks, D.H., Centeno, R., Culhane, J.L., Del Zanna, G., Doschek, G.A., Fletcher, L., Hara, H., Harra, L.K., Hillier, A.S., Imada, S., Klimchuk, J.A., Mariska, J.T., Pereira, T.M.D., Reeves, K.K., Sakao, T., Sakurai, T., Shimizu, T., Shimojo, M., Shiota, D., Solanki, S.K., Sterling, A.C., Su, Y., Suematsu, Y., Tarbell, T.D., Tiwari, S.K., Toriumi, S., Ugarte-Urra, I., Warren, H.P., Watanabe, T., Young, P.R.: 2019, Achievements of Hinode in the first eleven years. Publ. Astron. Soc. Japan 71, R1. DOI. ADS.

Hubbard, J., Goodrich, B., Wampler, S.: 2010, The ATST base: command-action-response in action. In: Radziwill, N.M., Bridger, A. (eds.) Software and Cyberinfrastructure for Astronomy, Proc. Soc. PhotoOpt. Instrum. Eng. (SPIE) CS-7740, 77402R. DOI. ADS.

Jess, D.B., Mathioudakis, M., Christian, D.J., Keenan, F.P., Ryans, R.S.I., Crockett, P.J.: 2010, ROSA: a high-cadence, synchronized multi-camera solar imaging system. Solar Phys. 261, 363. DOI. ADS.

Jess, D.B., Snow, B., Houston, S.J., Botha, G.J.J., Fleck, B., Krishna Prasad, S., Asensio Ramos, A., Morton, R.J., Keys, P.H., Jafarzadeh, S., Stangalini, M., Grant, S.D.T., Christian, D.J.: 2020, A chromospheric resonance cavity in a sunspot mapped with seismology. Nat. Astron. 4, 220. DOI. ADS.

Johansson, E.M., Goodrich, B.: 2012, Simultaneous control of multiple instruments at the Advanced Technology Solar Telescope. In: Radziwill, N.M., Chiozzi, G. (eds.) Software and Cyberinfrastructure for Astronomy II, Proc. Soc. Photo-Opt. Instrum. Eng. (SPIE) CS-8451, 84510J. DOI. ADS.

Keil, S.L., Rimmele, T., Keller, C.U., Hill, F., Radick, R.R., Oschmann, J.M., Warner, M., Dalrymple, N.E., Briggs, J., Hegwer, S.L., Ren, D.: 2003a, Design and development of the Advanced Technology Solar Telescope (ATST). In: Keil, S.L., Avakyan, S.V. (eds.) Innovative Telescopes and Instrumentation for Solar Astrophysics, Proc. Soc. Photo-Opt. Instrum. Eng. (SPIE) CS-4853, 240. DOI. ADS.

Keil, S., Rimmele, T., Keller, C., ATST Team: 2003b, Design and development of the Advanced Technology Solar Telescope. Astron. Nachr. 324, 303. DOI. ADS.

Kleint, L., Heinzel, P., Judge, P., Krucker, S.: 2016, Continuum enhancements in the ultraviolet, the visible and the infrared during the X1 flare on 2014 March 29. Astrophys. J. 816, 88. DOI. ADS.

Liu, Z., Xu, J., Gu, B.-Z., Wang, S., You, J.-Q., Shen, L.-X., Lu, R.-W., Jin, Z.-Y., Chen, L.-F., Lou, K., Li, Z., Liu, G.-Q., Xu, Z., Rao, C.-H., Hu, Q.-Q., Li, R.-F., Fu, H.-W., Wang, F., Bao, M.-X., Wu, M.-C., Zhang, B.-R.: 2014, New vacuum solar telescope and observations with high resolution. Res. Astron. Astrophys. 14, 705. DOI. ADS.

McBride, W.R., Wöger, F., Hegwer, S.L., Ferayorni, A., Gregory, B.S.: 2012, ATST visible broadband imager. In: McLean, I.S., Ramsay, S.K., Takami, H. (eds.) Ground-Based and Airborne Instrumentation for Astronomy IV, Proc. Soc. Photo-Opt. Instrum. Eng. (SPIE) CS-8446, 84461B. DOI. ADS.

Moll, R., Cameron, R.H., Schüssler, M.: 2011, Vortices in simulations of solar surface convection. Astron. Astrophys. 533, A126. DOI. ADS.

Neckel, H., Labs, D.: 1984, The solar radiation between 3300 and 12500 Å. Solar Phys. 90, 205. DOI. ADS.

Neckel, H., Labs, D.: 1994, Solar limb darkening 1986-1990 lambda 303 to 1099 nm. Solar Phys. $153,91$. DOI. ADS.

Peck, C.L., Wöger, F., Marino, J.: 2017, Influence of speckle image reconstruction on photometric precision for large solar telescopes. Astron. Astrophys. 607, A83. DOI. ADS.

Rast, M.P., Bello González, N., Bellot Rubio, L., Cao, W., Cauzzi, G., Deluca, E., de Pontieu, B., Fletcher, L., Gibson, S.E., Judge, P.G., Katsukawa, Y., Kazachenko, M.D., Khomenko, E., Landi, E., Martínez Pillet, V., Petrie, G.J.D., Qiu, J., Rachmeler, L.A., Rempel, M., Schmidt, W., Scullion, E., Sun, X., Welsch, B.T., Andretta, V., Antolin, P., Ayres, T.R., Balasubramaniam, K.S., Ballai, I., Berger, T.E., Bradshaw, S.J., Campbell, R.J., Carlsson, M., Casini, R., Centeno, R., Cranmer, S.R., Criscuoli, S., Deforest, C., Deng, Y., Erdélyi, R., Fedun, V., Fischer, C.E., González Manrique, S.J., Hahn, M., Harra, L., Henriques, V.M.J., Hurlburt, N.E., Jaeggli, S., Jafarzadeh, S., Jain, R., Jefferies, S.M., Keys, P.H., Kowalski, A.F., Kuckein, C., Kuhn, J.R., Kuridze, D., Liu, J., Liu, W., Longcope, D., Mathioudakis, M., McAteer, R.T.J., McIntosh, S.W., McKenzie, D.E., Miralles, M.P., Morton, R.J., Muglach, K., Nelson, C.J., Panesar, N.K., Parenti, S., Parnell, C.E., Poduval, B., Reardon, K.P., Reep, J.W., Schad, T.A., Schmit, D., Sharma, R., Socas-Navarro, H., Srivastava, A.K., Sterling, A.C., Suematsu, Y., Tarr, L.A., Tiwari, S., Tritschler, A., Verth, G., Vourlidas, A., Wang, H., Wang, Y.-M., NSO and DKIST Project, DKIST Instrument Scientists, DKIST Science Working Group, DKIST Critical Science Plan Community: 2021, Critical science plan for the Daniel K. Inouye Solar Telescope (DKIST). Solar Phys. 296, 70. DOI. ADS. 
Rempel, M.: 2012, Numerical sunspot models: robustness of photospheric velocity and magnetic field structure. Astrophys. J. 750, 62. DOI. ADS.

Rimmele, T., Keil, S.L., Keller, C., Hill, F., Penn, M., Goodrich, B., Hegwer, S., Hubbard, R., Oschmann, J., Warner, M., Dalrymple, N., Radick, R., ATST Team: 2003, Science objectives and technical challenges of the Advanced Technology Solar Telescope (invited review). In: Pevtsov, A.A., Uitenbroek, H. (eds.) Current Theoretical Models and Future High Resolution Solar Observations: Preparing for ATST, CS286, Astron. Soc. Pacific, San Francisco, 3. ADS.

Rimmele, T.R., Warner, M., Keil, S.L., Goode, P.R., Knölker, M., Kuhn, J.R., Rosner, R.R., McMullin, J.P., Casini, R., Lin, H., Wöger, F., von der Lühe, O., Tritschler, A., Davey, A., de Wijn, A., Elmore, D.F., Fehlmann, A., Harrington, D.M., Jaeggli, S.A., Rast, M.P., Schad, T.A., Schmidt, W., Mathioudakis, M., Mickey, D.L., Anan, T., Beck, C., Marshall, H.K., Jeffers, P.F., Oschmann, J.M., Beard, A., Berst, D.C., Cowan, B.A., Craig, S.C., Cross, E., Cummings, B.K., Donnelly, C., de Vanssay, J.-B., Eigenbrot, A.D., Ferayorni, A., Foster, C., Galapon, C.A., Gedrites, C., Gonzales, K., Goodrich, B.D., Gregory, B.S., Guzman, S.S., Guzzo, S., Hegwer, S., Hubbard, R.P., Hubbard, J.R., Johansson, E.M., Johnson, L.C., Liang, C., Liang, M., McQuillen, I., Mayer, C., Newman, K., Onodera, B., Phelps, L., Puentes, M.M., Richards, C., Rimmele, L.M., Sekulic, P., Shimko, S.R., Simison, B.E., Smith, B., Starman, E., Sueoka, S.R., Summers, R.T., Szabo, A., Szabo, L., Wampler, S.B., Williams, T.R., White, C.: 2020, The Daniel K. Inouye Solar Telescope - observatory overview. Solar Phys. 295, 172. DOI. ADS.

Scharmer, G.B., Bjelksjo, K., Korhonen, T.K., Lindberg, B., Petterson, B.: 2003, The 1-meter Swedish solar telescope. In: Keil, S.L., Avakyan, S.V. (eds.) Innovative Telescopes and Instrumentation for Solar Astrophysics, Proc. Soc. Photo-Opt. Instrum. Eng. (SPIE) CS-4853, 341. DOI. ADS.

Schlichenmaier, R., von der Lühe, O., Hoch, S., Soltau, D., Berkefeld, T., Schmidt, D., Schmidt, W., Denker, C., Balthasar, H., Hofmann, A., Strassmeier, K.G., Staude, J., Feller, A., Lagg, A., Solanki, S.K., Collados, M., Sigwarth, M., Volkmer, R., Waldmann, T., Kneer, F., Nicklas, H., Sobotka, M.: 2016, Active region fine structure observed at 0.08 arcsec resolution. Astron. Astrophys. 596, A7. DOI. ADS.

Sekulic, P., Gregory, S.B., Hegwer, S.L., Ferayorni, A., Woeger, F.: 2016, DKIST visible broadband imager alignment in laboratory: first results. In: Evans, C.J., Simard, L., Takami, H. (eds.) Ground-Based and Airborne Instrumentation for Astronomy VI, Proc. Soc. Photo-Opt. Instrum. Eng. (SPIE) CS-9908, 99085A. DOI. ADS.

Stein, R.F., Nordlund, Å.: 1998, Simulations of solar granulation. I. General properties. Astrophys. J. 499, 914. DOI. ADS

Stein, R.F., Nordlund, Å.: 2006, Solar small-scale magnetoconvection. Astrophys. J. 642, 1246. DOI. ADS.

Tritschler, A., Rimmele, T.R., Berukoff, S., Casini, R., Kuhn, J.R., Lin, H., Rast, M.P., McMullin, J.P., Schmidt, W., Wöger, F., DKIST Team: 2016, Daniel K. Inouye Solar Telescope: high-resolution observing of the dynamic Sun. Astron. Nachr. 337, 1064. DOI. ADS.

Tsiropoula, G., Tziotziou, K., Kontogiannis, I., Madjarska, M.S., Doyle, J.G., Suematsu, Y.: 2012, Solar fine-scale structures. I. Spicules and other small-scale, jet-like events at the chromospheric level: observations and physical parameters. Space Sci. Rev. 169, 181. DOI. ADS.

Vögler, A., Shelyag, S., Schüssler, M., Cattaneo, F., Emonet, T., Linde, T.: 2005, Simulations of magnetoconvection in the solar photosphere. Equations, methods, and results of the MURaM code. Astron. Astrophys. 429, 335. DOI. ADS.

von der Lühe, O.: 1984, Estimating Fried's parameter from a time series of an arbitrary resolved object imaged through atmospheric turbulence. J. Opt. Soc. Am. A 1, 510. DOI. ADS.

von der Lühe, O., Volkmer, R., Kentischer, T.J., Geißler, R.: 2012, The GREGOR broad-band imager. Astron. Nachr. 333, 894. DOI. ADS.

Wampler, S., Goodrich, B.: 2009, A scalable data handling system for ATST. In: Bohlender, D.A., Durand, D., Dowler, P. (eds.) Astronomical Data Analysis Software and Systems XVIII, CS-411, Astron. Soc. Pacific, San Francisco, 527. ADS.

Wang, J.Y., Markey, J.K.: 1978, Modal compensation of atmospheric turbulence phase distortion. J. Opt. Soc. Am. 68, 78. ADS.

Wedemeyer, S., Freytag, B., Steffen, M., Ludwig, H.-G., Holweger, H.: 2004, Numerical simulation of the three-dimensional structure and dynamics of the non-magnetic solar chromosphere. Astron. Astrophys. 414, 1121. DOI. ADS.

Wedemeyer-Böhm, S., Scullion, E., Steiner, O., Rouppe van der Voort, L., de La Cruz Rodriguez, J., Fedun, V., Erdélyi, R.: 2012, Magnetic tornadoes as energy channels into the solar corona. Nature 486, 505. DOI. ADS.

Wöger, F.: 2014, DKIST visible broadband imager interference filters. In: Ramsay, S.K., McLean, I.S., Takami, H. (eds.) Ground-Based and Airborne Instrumentation for Astronomy V, Proc. Soc. Photo-Opt. Instrum. Eng. (SPIE) CS-9147, 91479I. DOI. ADS.

Wöger, F., Ferayorni, A.: 2012, Accelerated speckle imaging with the ATST visible broadband imager. In: Radziwill, N.M., Chiozzi, G. (eds.) Software and Cyberinfrastructure for Astronomy II, Proc. Soc. Photo-Opt. Instrum. Eng. (SPIE) CS-8451, 84511C. DOI. ADS. 
Wöger, F., von der Lühe, O.: 2007, Field dependent amplitude calibration of adaptive optics supported solar speckle imaging. Appl. Opt. 46, 8015. DOI. ADS.

Wöger, F., von der Lühe, O., Reardon, K.: 2008, Speckle interferometry with adaptive optics corrected solar data. Astron. Astrophys. 488, 375. DOI. ADS.

Wöger, F., Uitenbroek, H., Tritschler, A., McBride, W., Elmore, D., Rimmele, T., Cowan, B., Wampler, S., Goodrich, B.: 2010, The ATST visible broadband imager: a case study for real-time image reconstruction and optimal data handling. In: McLean, I.S., Ramsay, S.K., Takami, H. (eds.) Ground-Based and Airborne Instrumentation for Astronomy III, Proc. Soc. Photo-Opt. Instrum. Eng. (SPIE) CS-7735, 773521. DOI. ADS.

Wöger, F., McBride, W., Ferayorni, A., Gregory, S., Hegwer, S., Tritschler, A., Uitenbroek, H.: 2012, The visible broadband imager: the Sun at high spatial and temporal resolution. In: Rimmele, T.R., Tritschler, A., Wöger, F., Collados Vera, M., Socas-Navarro, H., Schlichenmaier, R., Carlsson, M., Berger, T., Cadavid, A., Gilbert, P.R., Goode, P.R., Knölker, M. (eds.) Second ATST-EAST Meeting: Magnetic Fields from the Photosphere to the Corona, CS-463, Astron. Soc. Pacific, San Francisco, 431. ADS.

Yadav, N., Cameron, R.H., Solanki, S.K.: 2021, Vortex flow properties in simulations of solar plage region: evidence for their role in chromospheric heating. Astron. Astrophys. 645, A3. DOI. ADS.

Publisher's Note Springer Nature remains neutral with regard to jurisdictional claims in published maps and institutional affiliations.

\section{Authors and Affiliations}

\section{Friedrich Wöger ${ }^{1} \cdot$ Thomas Rimmele $^{1}$ (D) Andrew Ferayorni ${ }^{1} \cdot$ Andrew Beard $^{1}$. Brian S. Gregory ${ }^{1} \cdot$ Predrag Sekulic $^{1,2}$. Steven L. Hegwer ${ }^{1}$}

F. Wöger

fwoeger@nso.edu

T. Rimmele

trimmele@nso.edu

A. Ferayorni

andyf@nso.edu

A. Beard

andyb@nso.edu

B.S. Gregory

sgregory@nso.edu

P. Sekulic

psekulic@nso.edu

1 National Solar Observatory, 3665 Discovery Drive, Boulder, CO 80303, USA

2 KBR/NASA Goddard Space Flight Center, 8800 Greenbelt Road, Greenbelt, MD 20771, USA 